\title{
Structural health monitoring and damage detection using an intelligent parameter varying (IPV) technique
}

\author{
Soheil Saadat ${ }^{\mathrm{a}, *}$, Mohammad N. Nooria ${ }^{\mathrm{a}}$, Gregory D. Buckner ${ }^{\mathrm{a}}$, Tadatoshi Furukawa ${ }^{\mathrm{b}}$, \\ Yoshiyuki Suzukic \\ ${ }^{a}$ Department of Mechanical and Aerospace Engineering, North Carolina State University, Raleigh, NC 27695-7910 NC, USA \\ ${ }^{\mathrm{b}}$ Department of Global Architecture, Graduate School of Engineering, Osaka University, 2-I Yamadaoka, Suita, \\ Osaka 565-0871, Japan \\ ${ }^{\mathrm{c} D i s a s t e r}$ Prevention Research Institute, Kyoto University, Gokasho, Uji, Kyoto 611-0011, Japan
}

\begin{abstract}
Most structural health monitoring and damage detection strategies utilize dynamic response information to identify the existence, location, and magnitude of damage. Traditional model-based techniques seek to identify parametric changes in a linear dynamic model, while non-model-based techniques focus on changes in the temporal and frequency characteristics of the system response. Because restoring forces in base-excited structures can exhibit highly non-linear characteristics, non-linear model-based approaches may be better suited for reliable health monitoring and damage detection. This paper presents the application of a novel intelligent parameter varying (IPV) modeling and system identification technique, developed by the authors, to detect damage in base-excited structures. This IPV technique overcomes specific limitations of traditional model-based and non-model-based approaches, as demonstrated through comparative simulations with wavelet analysis methods. These simulations confirm the effectiveness of the IPV technique, and show that performance is not compromised by the introduction of realistic structural non-linearities and ground excitation characteristics.
\end{abstract}

(C) 2004 Elsevier Ltd. All rights reserved.

Keywords: System identification; Artificial neural networks; Hysteresis; Non-linear systems; Damage detection; Structural health monitoring; Wavelet analysis

\section{Introduction}

Civil structures, particularly those subject to seismic excitation, are prone to damage and deterioration

\footnotetext{
* Corresponding author. Tel.: +1-919-515-5260; Fax: +1-919515-7968.

E-mail addresses: ssaadat@eos.ncsu.edu (S. Saadat), mnoori@eos.ncsu.edu (M.N. Noori), gbuckner@eos.ncsu.edu (G.D. Buckner), furukawa@ga.eng.osaka-u.ac.jp (T. Furukawa), suzuki@zeisei.dpri.kyoto-u.ac.jp (Y. Suzuki).
}

during their service lives. To ensure structural integrity it is desirable to monitor these structures to detect the existence, location, and severity of any damage in real time. Common health monitoring and damage detection practices involve systematic visual inspections by experienced engineers who determine the location and extent of damaged zones. If these damaged zones are readily accessible, various experimental techniques can be used to assess the location and severity of damage with greater 
precision. The ever-increasing complexity of civil structures makes the practicality and reliability of such manual approaches questionable, particularly following natural disasters like earthquakes. For this reason, the development of reliable monitoring techniques has received increasing attention over the last decade.

Health monitoring and damage detection techniques can be classified according to either their detection capabilities (global techniques merely infer the existence of damage, while local techniques assist in locating it) or based on the extent of prior knowledge required (model-based techniques utilized explicit mathematical descriptions of the system dynamics, while non-model-based techniques rely on signal processing of measured responses). Both model-based and non-model-based techniques have been successfully demonstrated for damage detection in structural applications. Model-based approaches typically rely on parametric system identification using linear, time-invariant models. Non-model-based alternatives include modal analysis, dynamic flexibility measurements, matrix update methods, and wavelet transform techniques. These methods typically seek to identify damage from changes in structural vibration characteristics (response measurements, natural frequencies, mode shapes, etc.). Excellent surveys may be found in Refs. [1-4].

In recent years, there has been increasing interest in the use of artificial neural networks for both model-based and non-model-based damage detection approaches. Artificial neural networks are typically utilized in one of the two ways. The pattern recognition capabilities of neural networks allow the identification of damage using response measurements from damaged and undamaged structures (non-model-based approaches) $[5,6]$. Alternately, the system identification capabilities of neural networks enable the estimation of dynamic parameters such as stiffness, mass, and damping (model-based approaches). Most of the published research involving structural system identification has focused on parametric modeling and system identification using linear, time-invariant models. However, because of their unique capabilities in non-linear function approximation [7], artificial neural networks have also been used for non-parametric modeling and system identification (non-model-based, or "black box" approaches). The literature abounds with "black box" implementations of artificial neural networks for non-parametric modeling, identification, and control of non-linear dynamic systems $[8,9]$ and health monitoring and damage detection $[10,11]$.

\section{Health monitoring and damage detection using the intelligent parameter varying technique}

Neural network approaches typically involve input-output training to predict the dynamic response of a "healthy" structure to known input excitations. This predicted response is compared to the response of the same damaged structure to infer information about the presence, location, and extent of damage. Such methodologies, however, may fail to detect authentic damage if the response of the damaged structure moves beyond the representative domain of the trained neural network. Additionally, few researchers have addressed the complexities of detecting damage in structural components with elasto-plastic and hysteretic restoring force characteristics.

This paper demonstrates the intelligent parameter varying (IPV) modeling and identification technique [12] for damage detection in non-linear structures subject to seismic excitation. This unique approach to non-linear system identification combines the advantages of parametric models with the non-parametric capabilities of artificial neural networks. It incorporates radial basis function networks (RBFN) into a traditional parametric model to identify the non-linear, time-varying portions of the system dynamics, in this case inelastic and hysteretic restoring forces that would be very difficult to model using traditional approaches [12]. Parametric system identification approaches require accurate, a priori representations of system non-linearities to obtain an optimal models. The IPV approach provides functional representations of system non-linearities without prior knowledge of their constitutive characteristics.

The IPV technique reveals the evolution of damage through the identification of structural restoring forces, rather than comparing response characteristics to a "healthy" reference state. Contrary to neural network techniques that require interstorey relative velocities and displacements, the IPV technique uses recorded inter-storey relative 


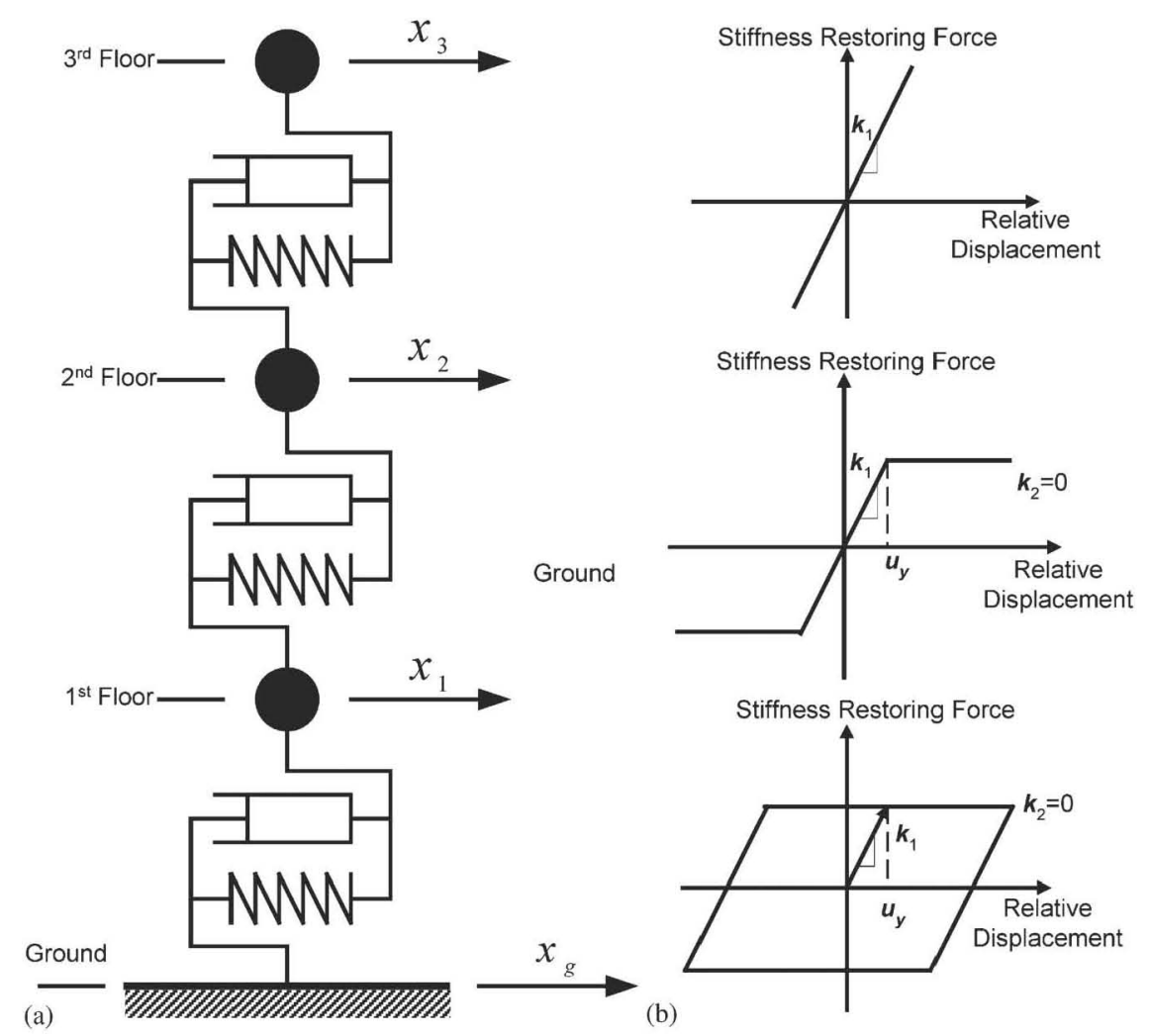

Fig. 1. (a) Lumped-mass model of the three-storey shear-building, (b) restoring force models used for response simulations.

accelerations as network inputs, avoiding the challenges associated with integrating acceleration responses.

The performance of this IPV approach in determining the existence, location, and extent of structural damage is compared to a wavelet analysis approach. Wavelet analysis techniques, which have been extensively used for structural health monitoring and damage detection in recent years [13,14], decompose quasi-stationary and non-stationary signals into linear combinations of time-frequency and time-scale wavelets [15]. Continuous wavelet transforms provide two-dimensional time-frequency maps of one-dimensional time-domain signals, whereas discrete wavelet transforms decompose the signal into low- and high-frequency components otherwise known as approximation and detail levels, respectively. Simulations using realistic non-linear structures and measured earthquake ground accelerations reveal the benefits of the IPV approach in identifying the existence, location, time of occurrence, and magnitude of structural damage.

\section{System modeling}

The effectiveness of health monitoring and damage detection strategies for multi-storey buildings subjected to seismic excitations can be assessed using a simple shear-building model. Such a model can be constructed by assuming that masses are lumped at each floor, and that each floor is constrained to move laterally. Fig. 1a shows the three-storey shear-building model used for this research. Note that each lumped mass $m_{i}$ represents the collective mass of the floor and its associated columns and beams, and that the springs and dampers represent the collective structural stiffness and damping between adjacent floors. Resulting lateral floor displacements represent the building's degrees of freedom and are represented by the state vector $\mathbf{x}=\left[x_{g}, x_{1}, x_{2}, x_{3}\right]^{\mathrm{T}}$. 
In accordance with Newton's 2nd law, the lateral equations of motion can be expressed as

$-f_{3}-c_{3}\left(\dot{x}_{3}-\dot{x}_{2}\right)=m_{3} \ddot{x}_{3}$,

$-f_{2}-c_{2}\left(\dot{x}_{2}-\dot{x}_{1}\right)+f_{3}+c_{3}\left(\dot{x}_{3}-\dot{x}_{2}\right)=m_{2} \ddot{x}_{2}$,

$-f_{1}-c_{1}\left(\dot{x}_{1}-\dot{x}_{g}\right)+f_{2}+c_{2}\left(\dot{x}_{2}-\dot{x}_{1}\right)=m_{1} \ddot{x}_{1}$,

where $m_{1}, m_{2}, m_{3}$ represent the lumped masses, $c_{1}, c_{2}, c_{3}$ are constant structural damping coefficients, and $f_{1}, f_{2}, f_{3}$ are the inelastic stiffness restoring forces of the building. Alternately, these state equations can be expressed in terms of storey drifts $u_{1}, u_{2}, u_{3}$

$$
\begin{aligned}
& -f_{3}-c_{3}\left(\dot{u}_{3}-\dot{u}_{2}\right)-m_{3} \ddot{x}_{g}=m_{3} \ddot{u}_{3}, \\
& -f_{2}-c_{2}\left(\dot{u}_{2}-\dot{u}_{1}\right)+f_{3} \\
& \quad+c_{3}\left(\dot{u}_{3}-\dot{u}_{2}\right)-m_{2} \ddot{x}_{g}=m_{2} \ddot{u}_{2}, \\
& -f_{1}-c_{1}\left(\dot{u}_{1}-\dot{u}_{g}\right)+f_{2} \\
& \quad+c_{2}\left(\dot{u}_{2}-\dot{u}_{1}\right)-m_{1} \ddot{x}_{g}=m_{1} \ddot{u}_{1},
\end{aligned}
$$

where

$u_{3}=x_{3}-x_{g}, \quad u_{2}=x_{2}-x_{g}, \quad u_{1}=x_{1}-x_{g}$.

Eq. (2) can be expressed in matrix form as

$\mathbf{M u ̈}+\mathbf{C u}=-\mathbf{M} \ddot{\mathbf{x}}_{g}-\mathbf{f}(\mathbf{x}, \mathbf{u})$,

where $\mathbf{M}$ and $\mathbf{C}$ are the diagonal mass and coupled damping matrices, respectively,

$\mathbf{M}=\left[\begin{array}{ccc}m_{1} & 0 & 0 \\ 0 & m_{2} & 0 \\ 0 & 0 & m_{3}\end{array}\right]$,
$\mathbf{C}=\left[\begin{array}{ccc}c_{1}+c_{2} & -c_{2} & 0 \\ -c_{2} & c_{2}+c_{3} & -c_{3} \\ 0 & -c_{3} & c_{3}\end{array}\right]$.

\section{Simulations}

In Section 4.1, simulated acceleration responses of a three-storey shear-building model subject to seismic excitation are presented. Realistic structural damage is introduced to facilitate the comparison of health monitoring and damage detection algorithms. In Section 4.2, a popular discrete wavelet analysis technique is applied to detect structural damage using the acceleration responses. In Section 4.3, IPV modeling and system identification is used for the same purpose.

\subsection{Structural response simulations}

To evaluate the performance of wavelet analysis and IPV techniques for structural damage detection, a series of simulations was conducted using the three-storey shear-building model (1). Three distinct restoring force models (elastic, elasto-plastic, and hysteretic) were considered, as shown in Fig. 1 b. The model's dynamic parameters were selected to provide typical natural frequencies for a three-storey building. Primary and secondary column stiffnesses of $2500 \mathrm{~N} / \mathrm{m}$ and $0 \mathrm{~N} / \mathrm{m}$ were selected for each floor, floor masses were set to $1.0 \mathrm{~kg}$. Structural damping was neglected to simplify the comparison of damage detection techniques, though it could easily be incorporated into the simulations. Measured ground accelerations from the El Centro 1940 earthquake and $3-\mathrm{Hz}$ sinusoids were used as seismic excitations for these simulations and building response data was generated using the Newmark linear acceleration integration algorithm [16]. The integration time-step $(0.002 \mathrm{~s})$ was selected to be one-tenth the sampling period of the excitation, allowing the integration algorithm to accurately detect instances of yield and recovery for the elasto-plastic and hysteretic restoring force models shown in Fig. 1b.

Structural damage was simulated using two different mechanisms. In the first, damage to a given floor was simulated as being a $10 \%$ reduction in primary column stiffness that occurred when the relative floor displacement exceeded $80 \%$ of the corresponding yield displacement $\left(0.16 \mathrm{e}^{-3} \mathrm{~m}\right)$. Subsequent relative floor displacements exceeding the same threshold resulted in an additional $10 \%$ reduction in primary column stiffness. This damage mechanism was introduced to elastic, elasto-plastic, and hysteretic restoring force models. When applied to elasto-plastic and hysteretic models the yield displacements were not changed, but the restoring forces associated with the yield displacements were reduced. 
Table 1

Simulation parameters for Cases I-VIII

\begin{tabular}{|c|c|c|c|}
\hline $\begin{array}{l}\text { Simulation } \\
\text { Case }\end{array}$ & $\begin{array}{l}\text { Base } \\
\text { excitation }\end{array}$ & $\begin{array}{l}\text { Restoring } \\
\text { force model }\end{array}$ & Damage Mechanism \\
\hline I & $\begin{array}{l}\text { 3-Hz } \\
\text { Sinusoid }\end{array}$ & Elastic & 1st mechanism \\
\hline II & $\begin{array}{l}\text { E1 Centro } \\
1940\end{array}$ & Elastic & 1st mechanism \\
\hline III & $\begin{array}{l}3-\mathrm{Hz} \\
\text { Sinusoid }\end{array}$ & Elastic & $\begin{array}{l}\text { 2nd mechanism } \\
\text { followed by the } \\
\text { 1st mechanism }\end{array}$ \\
\hline IV & $\begin{array}{l}\text { El Centro } \\
1940\end{array}$ & Elastic & $\begin{array}{l}\text { 2nd mechanism } \\
\text { followed by the } \\
1 \text { st mechanism }\end{array}$ \\
\hline V & $\begin{array}{l}\text { 3-Hz } \\
\text { Sinusoid }\end{array}$ & Elasto-plastic & 1st mechanism \\
\hline VI & $\begin{array}{l}\text { El Centro } \\
1940\end{array}$ & Elasto-plastic & 1st mechanism \\
\hline VII & $\begin{array}{l}\text { 3-Hz Sinusoid } \\
\text { Sinusoid }\end{array}$ & Hysteretic & 1st mechanism \\
\hline VIII & $\begin{array}{l}\text { El Centro } \\
1940\end{array}$ & Hysteretic & 1st mechanism \\
\hline
\end{tabular}

In the second mechanism, damage to a given floor was simulated as change of restoring force model from elastic to elasto-plastic when the relative floor displacement exceeded $0.16 \mathrm{e}^{-3} \mathrm{~m}$. The yield displacement of the new elasto-plastic model was set to $0.2 \mathrm{e}^{-3} \mathrm{~m}$. Subsequent relative floor displacements exceeding $80 \%$ of the corresponding yield displacement $\left(0.16 \mathrm{e}^{-3} \mathrm{~m}\right)$ resulted in an additional $20 \%$ reduction in primary column stiffness. This damage mechanism was utilized only for the elastic restoring force model.

Based on the restoring force models, seismic inputs, and damage mechanisms described above, a total of eight simulation cases were considered, as summarized in Table 1. In all cases, damage was restricted from occurring during the first $4.0 \mathrm{~s}$ of each simulation, and subsequent damage was restricted from occurring within $10.0 \mathrm{~s}$ of initial damage. Furthermore, to simplify the interpretation of results, the number of damage occurrences was limited to two per floor. Note that the occurrence and magnitude of damage for each floor was not necessarily coincident with other floors. Representative acceleration responses from two of these simulation cases, Cases IV and VIII, are presented in Fig. 2.

One may argue that the presence, location, and time of damage can be detected visually from acceleration response plots and that there is no need for sophis- ticated techniques such as IPV or wavelet analysis. While this may be true for low-order linear models with simple harmonic inputs (Simulation Case I: elastic restoring force model with $3 \mathrm{~Hz}$ sinusoidal excitation), for "realistic" structures with inelastic and hysteretic behavior subject to actual seismic excitations, both visual inspection and traditional damage detection may fail to detect structural damage. The intent of these simulations is to demonstrate the improved effectiveness of the IPV technique as more realistic effects are considered.

\subsection{Wavelet analysis for health monitoring and damage detection}

Damage detection techniques based on wavelet analysis typically utilize measured structural responses and follow one of two approaches. In the first approach, discrete wavelet transforms are tuned to detect abrupt changes in the response by decomposing the signal into approximation and detail levels. "Spikes" in detail level decompositions correspond to abrupt changes in the response that might be associated with structural damage. In the second approach, continuous wavelet transforms detect changes in the structure's natural frequencies by generating a timefrequency map of the response signal.

In this study Daubechies II analyzing wavelets were implemented using MATLAB's wavelet analysis toolbox [15]. The acceleration responses of Fig. 2 were decomposed into one approximation and three detail levels, where the first detail level corresponds to the highest frequency content. Fig. 3 shows the first detail level (D1) of the corresponding discrete wavelet transforms (DWT).

Fig. 3a shows that, for simulation Case IV, distinct spikes at approximately $4.0 \mathrm{~s}$ match closely with initial damage occurrence times. However, once the restoring force model changes from elastic to elastoplastic, repeated transitions from elastic to plastic regions create similar spikes at this detail level that might incorrectly be identified as (or mask the occurance of) subsequent damage. For this reason, accurate detection of subsequent damage (at $14.514 \mathrm{~s}$, $14.618 \mathrm{~s}$, and $14.016 \mathrm{~s}$ on the building's first, second, and third floors, respectively) is not possible. Fig. $3 \mathrm{~b}$ shows that, for simulation Case VIII, spikes at this detail level do not correspond to occurrences of 

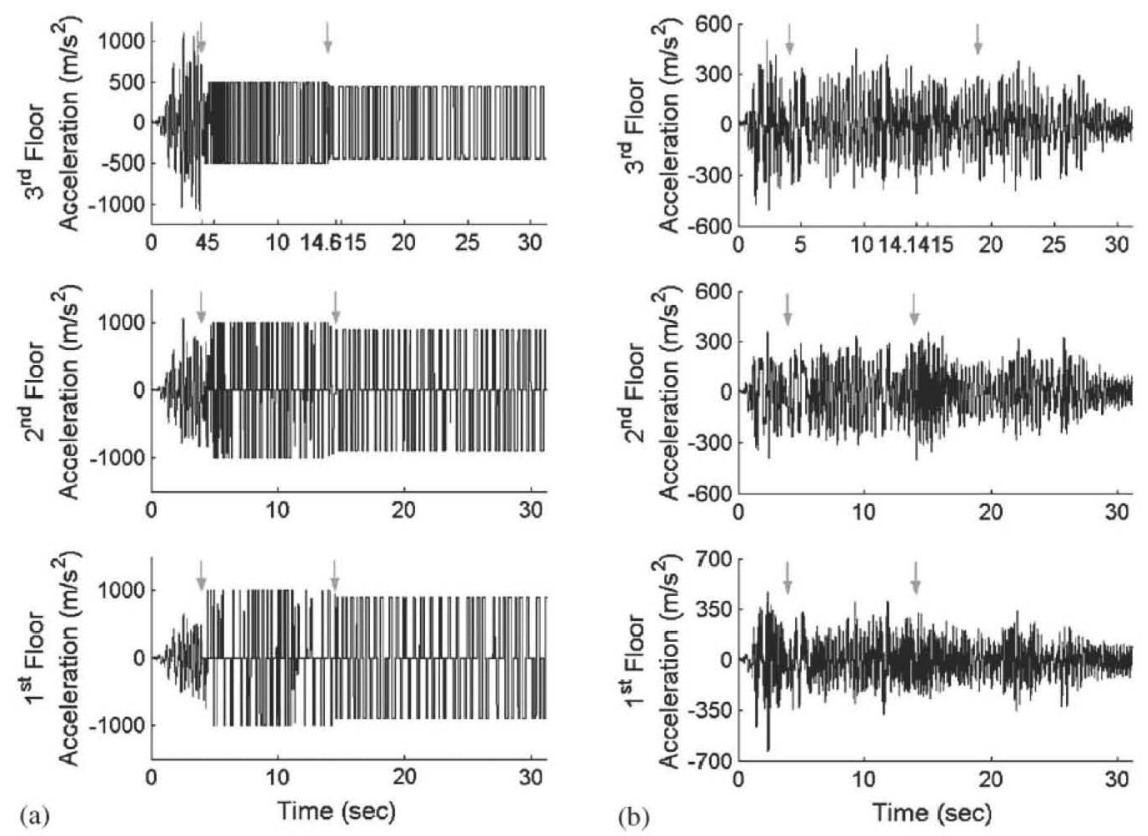

Fig. 2. Acceleration responses $\left(\mathrm{m} / \mathrm{s}^{2}\right.$ ), with damage occurrences indicated by arrows, (a) simulation Case IV, (b) simulation Case VIII.
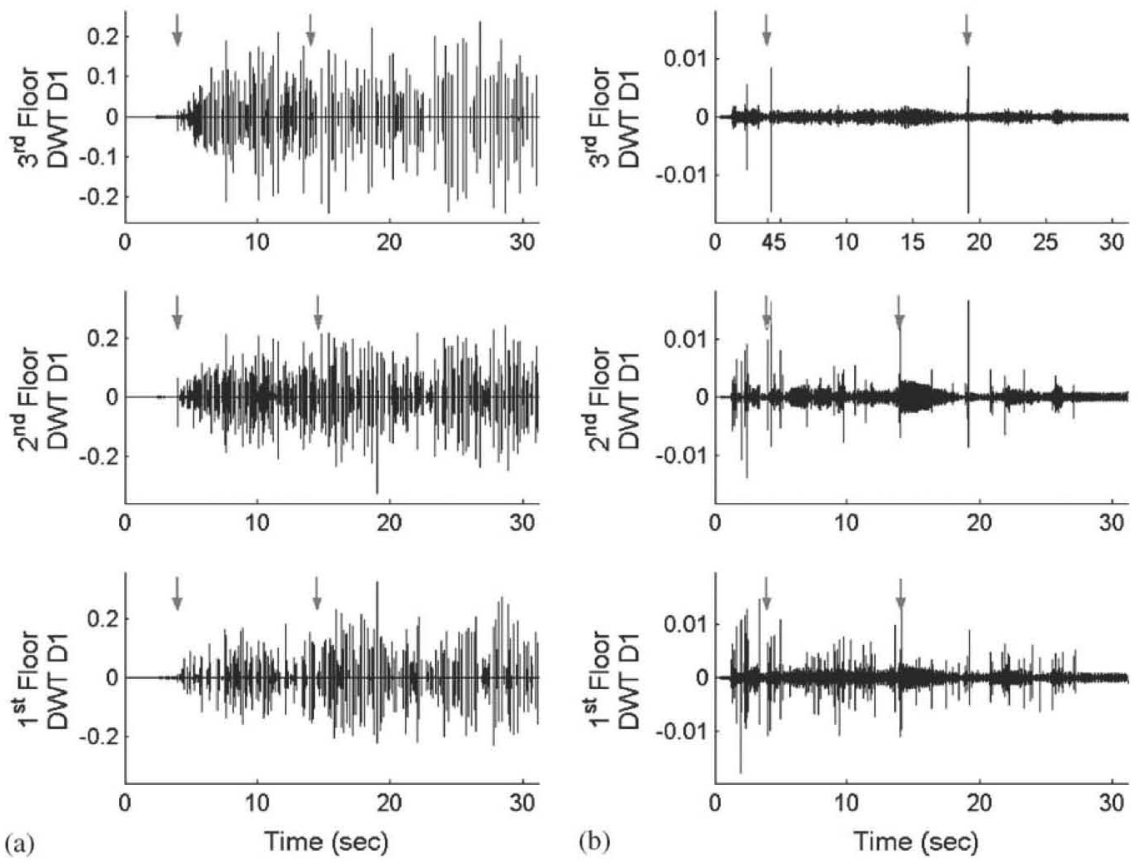

Fig. 3. Wavelet analysis, with damage occurrences indicated by arrows, (a) simulation Case IV, (b) simulation Case VIII. 
structural damage (at $14.142 \mathrm{~s}, 14.004 \mathrm{~s}$, and $19.006 \mathrm{~s}$ on the building's first, second, and third floors, respectively) and thus cannot be used for detection. Similar trends were observed in simulation Cases I, II, III, V, VI, and VII. These results suggest that the performance of wavelet analysis for structural damage detection depends strongly on the excitation and restoring force characteristics. Clearly, the performance deteriorates as more realistic seismic excitation and nonlinear restoring forces are introduced.

\subsection{IPV technique for health monitoring and damage detection}

The IPV modeling and system identification technique [12] was similarly applied to the acceleration responses of simulation Cases I-VIII. As mentioned in Section 2, the IPV technique incorporates radial basis function networks (RBFN) into a parametric model to identify the structural restoring forces. For the three-storey shear-building model (1), the stiffness and damping terms were combined into net restoring forces $R_{1}, R_{2}, R_{3}$ :

$-R_{3}-m_{3} \ddot{x}_{g}=m_{3} \ddot{u}_{3}$,

$-R_{2}+R_{3}-m_{2} \ddot{x}_{g}=m_{2} \ddot{u}_{2}$,

$-R_{1}+R_{2}-m_{1} \ddot{x}_{g}=m_{1} \ddot{u}_{1}$.

Three separate RBFN networks $g_{1}, g_{2}, g_{3}$ were used to model these restoring forces:

$\hat{R}_{3}=g_{3}\left(\ddot{u}_{3}, \ddot{x}_{g}\right)$,

$\hat{R}_{2}=g_{2}\left(\ddot{u}_{2}, \ddot{x}_{g}, \hat{R}_{3}\right)$,

$\hat{R}_{1}=g_{1}\left(\ddot{u}_{1}, \ddot{x}_{g}, \hat{R}_{2}\right)$.

This equation can be rearranged to provide predicted accelerations for each floor:

$\hat{\ddot{u}}_{3}=\frac{-\hat{R}_{3}-m_{3} \ddot{x}_{g}}{m_{3}}$,

$\hat{\ddot{u}}_{2}=\frac{-\hat{R}_{2}+\hat{R}_{3}-m_{2} \ddot{x}_{g}}{m_{2}}$,

$\hat{\ddot{u}}_{1}=\frac{-\hat{R}_{1}+\hat{R}_{2}-m_{1} \ddot{x}_{g}}{m_{1}}$.

Fig. 4 shows the specific architecture of the RBFNs used. For a single-output RBFN with $N$ hidden layer
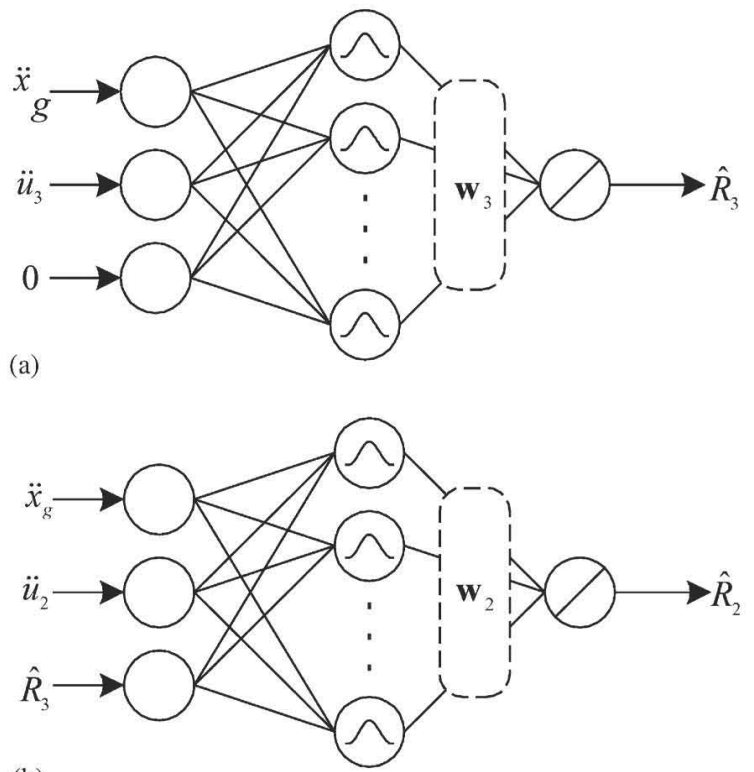

(b)

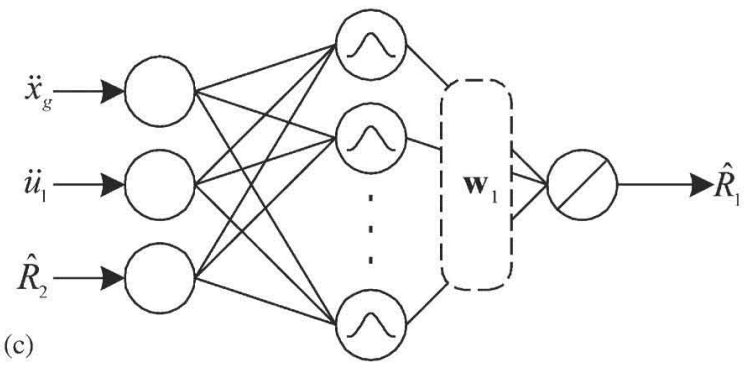

Fig. 4. Architecture of RBF networks used for modeling restoring forces, (a) 3rd, (b) 2nd, and (c) 1st Floor.

neurons, there are three parameters that determine the network output: the network weights $\boldsymbol{w}$ (an $N$-element vector), the neuron centers $\boldsymbol{c}$ (an $N$-element vector), and the neuron spread $s$ (an $N$-element vector, for this application a scalar). Specifying the "best" parameters for a given estimation problem constitutes a non-linear optimization process with potentially large numbers of local minima. However, by specifying "reasonable" neuron centers $c$ and spreads $s$ (based on known ranges of recorded input/output data), the process of specifying the "best" network weight vector $\boldsymbol{w}$ constitutes a linear optimization problem with only one global minimum for a given error cost function [17]. For this application, a quadratic cost function of prediction errors (defined as the difference between 

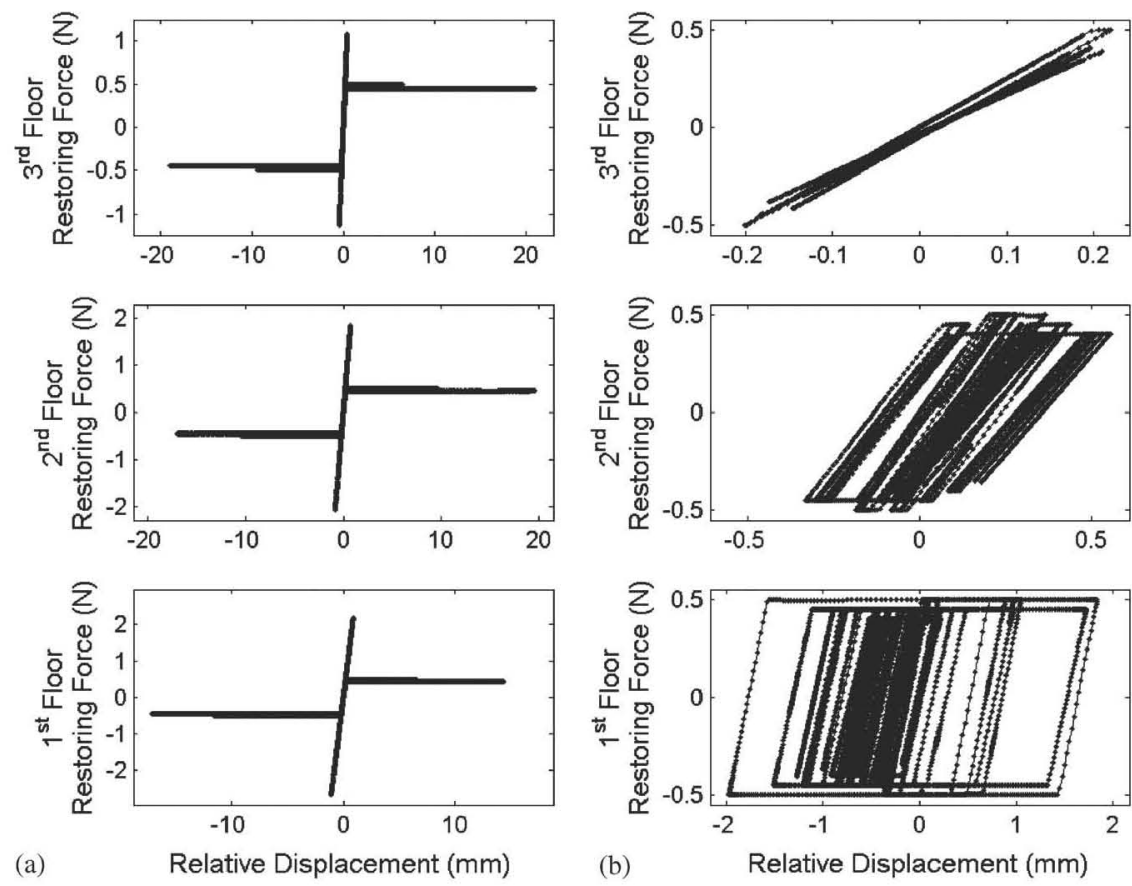

Fig. 5. Estimated (•) and simulated (-) net restoring forces (N), (a) simulation Case IV (b) simulation Case VIII.

the recorded and estimated acceleration response of the structure) was used.

Because the inputs to each RBFN (Fig. 4) have different ranges, each input was initially normalized based on its minimum and maximum values. Three basis functions were uniformly distributed along normalized dimensions of each input (with centers located at $-0.25,0.50$, and 1.25 ), resulting in 27 basis functions for each RBFN. The spread of each basis function was specified to be 5.0 (approximately three times the largest distance between basis functions).

Recall that the IPV technique presented here identifies structural restoring forces and damage mechanisms without a priori knowledge or assumptions regarding their constitutive characteristics. Because the identified restoring forces are represented by and stored in its network weights, all network weights $w$ were initialized to zero.

To ensure that the RBFNs properly generalized information from the simulated acceleration responses, for each simulation case the data was divided into training and testing (validation) sets. Each $31.178 \mathrm{~s}$ simulation case consisted of 15590 time samples of ground acceleration and resulting floor accelerations. Each training data set consisted of 7795 time samples (one half of the simulated time history), selected randomly from the simulation data. The remaining 7795 time samples were randomly ordered and used to construct the testing (validation) data set.

A standard backpropagation of error training algorithm, based on a quadratic error cost function, was implemented for training [7]. This training algorithm was implemented in the following systematic manner. First, acceleration data from the building's base and third floor were used to estimate the net restoring force on the third floor $\hat{R}_{3}=g_{3}\left(\ddot{u}_{3}, \ddot{x}_{g}\right)$. Next, this predicted restoring force, combined with acceleration data from the building's base, second and third floors, was used to estimate the net restoring force on the second floor $\hat{R}_{2}=g_{2}\left(\ddot{u}_{2}, \ddot{x}_{g}, \hat{R}_{3}\right)$. Finally, this predicted restoring force, combined with acceleration data from the building's base, first and second floors, was used to estimate the net restoring force on the first floor $\hat{R}_{1}=g_{1}\left(\ddot{u}_{1}, \ddot{x}_{g}, \hat{R}_{2}\right)$.

Training continued until the error cost function (evaluated based on prediction errors from the testing 

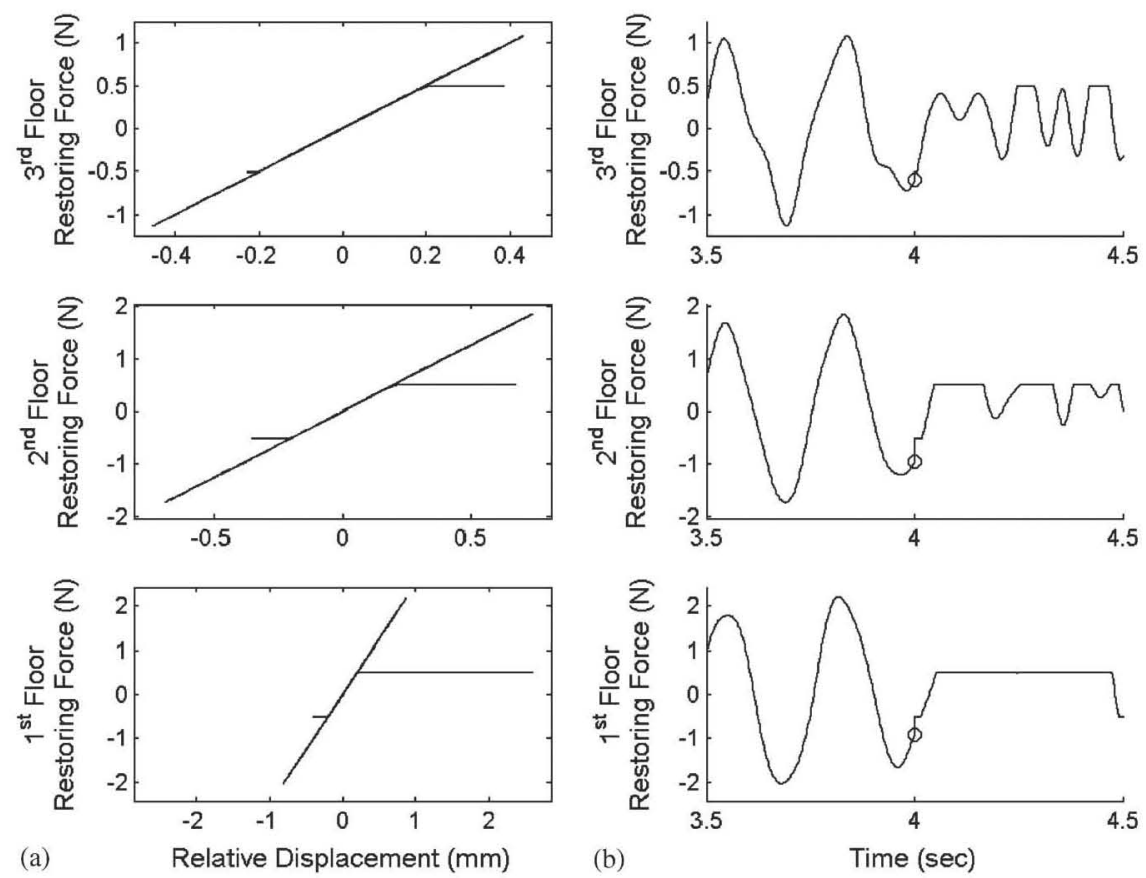

Fig. 6. Snapshots of estimated restoring forces $(N)$ for simulation Case IV, (a) vs. relative displacement (b) vs. time, before and after damage (o).

data set) fell below $1 \%$ for two consecutive training epochs. A training epoch constitutes one complete presentation of the training data set to modify (update) the network weight vectors $\boldsymbol{w}$. Typical "identified" restoring forces $\hat{R}_{1}, \hat{R}_{2}, \hat{R}_{3}$ and "simulated" restoring forces are presented in Fig. 5. Fig. 5a shows that, for simulation Case IV, the force-displacement characteristics change from elastic to elasto-plastic. In this case, structural damage results in severe softening and plastic deformation of columns at relative displacements exceeding $\pm 0.2 \mathrm{e}^{-3} \mathrm{~m}$. Fig. $5 \mathrm{~b}$ shows that, for simulation Case VIII, the force-displacement characteristics remain hysteretic even after the occurrence of structural damage. As before, recurring softening of the primary column stiffnesses results in lower restoring forces and consequent reductions in energy dissipation capacity during plastic deformation. It is important to note that the IPV technique identified these net restoring forces without a priori knowledge of their characterization (elastic, elasto-plastic, or hysteretic): the initial RBFN weights were set to zero (not distributed about the "actual" values).
To precisely isolate and identify the occurrence of damage, this IPV technique was implemented in a "snapshot mode", where response data were divided into 1-s time intervals and restoring forces were identified. Using this approach, changes in restoring force characteristics could be readily identified. Figs. 6 and 7 show selected restoring force snapshots for simulation Cases IV and VIII, and clearly reveal the occurrence of structural damage. Fig. 6 shows a 1-s snapshot associated with the first occurrence of structural damage for simulation Case IV (3.5-4.5 s). The changes in restoring force characteristics, from elastic to elasto-plastic, are clearly evident in Fig. 6a. Similarly, the time of occurrence $(4.002 \mathrm{~s})$ is readily determined from Fig. $6 \mathrm{~b}$. Fig. 7 isolates the changes in restoring force characteristics for simulation Case VIII (14.0-15.0 s). Fig. 7a shows that primary column stiffnesses are already reduced due to previous structural damage, resulting in lower restoring forces during plastic deformation (from $0.5 \mathrm{~N}$ to around $0.4 \mathrm{~N}$ ). Furthermore, this figure shows additional softening resulting from structural damage at 14.004 and $14.142 \mathrm{~s}$, as shown in Fig. 7b. 

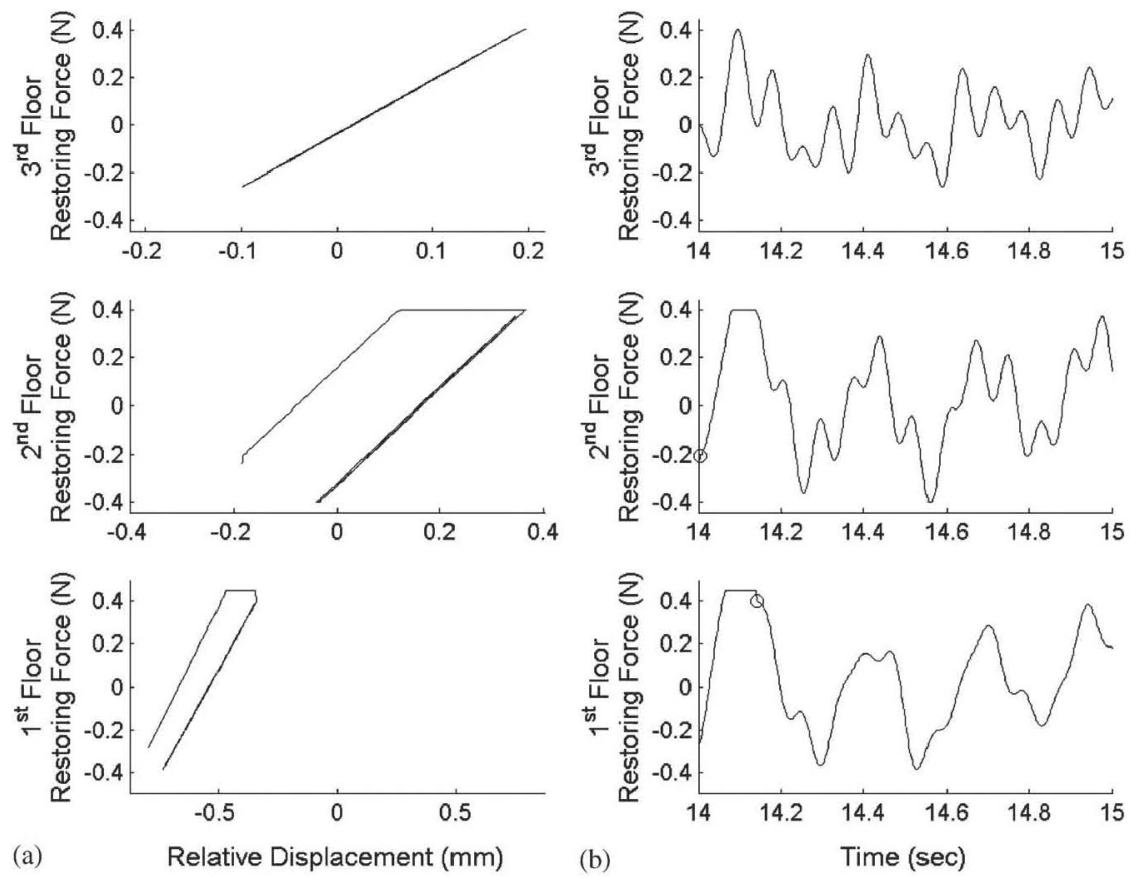

Fig. 7. Snapshots of estimated restoring forces $(N)$ for simulation Case VIII, (a) vs. relative displacement (b) vs. time, before and after damage (o).

The results presented here clearly demonstrate the unique capabilities of IPV modeling and system identification to identify the existence, location, time of occurrence, and magnitude of structural damage, even in non-linear structures.

\section{Conclusion}

A simple yet powerful technique that overcomes the intrinsic limitations of parametric and non-parametric techniques, while preserving unique benefits of each, is presented. Simulations reveal that this intelligent parameter varying (IPV) technique effectively identifies the inelastic restoring forces without a priori knowledge of their constitutive characteristics and can be used to detect the presence, location, and magnitude of damage in the structure. Unlike popular wavelet analysis techniques, the performance of the IPV technique is not affected by structural non-linearities or excitation characteristics.

The simplicity of this IPV technique, combined with its adaptive capabilities to approximate and general- ize non-linear information, makes it ideally suited to health monitoring and damage detection applications as shown in this study. Effects of measurement noise and incomplete data on its performance are issues that will be addressed in subsequent studies.

\section{Acknowledgements}

The research reported in this paper was conducted within the framework of United StatesJapan Cooperative Research on Smart Structures and Structural Health Monitoring with Disaster Prevention Research Institute of Japan, and was supported in part by grants from the National Institute of Aerospace, and RJ Reynolds Endowment Funds.

\section{References}

[1] S.W. Doebling, C.R. Farrar, M.B. Prime, D.W. Shevitz, Damage Identification and Health Monitoring of Structural and Mechanical Systems from Changes in Their Vibration 
Characteristics: A Literature Review, Los Alamos National Laboratory, Los Alamos, NM, LA-13070-MS, 1996.

[2] S.W. Doebling, C.R. Farrar, M.B. Prime, D.W. Shevitz, A review of damage identification methods that examine changes in dynamic properties, Shock Vib. Dig. 30 (1998) 91-105.

[3] M. Noori, F.-G. Yuan, S. Saadat, Z. Hou, A. Hera, A. Masuda, A. Sone, Recent and on-going efforts in structural health monitoring, in: K. Kawashima, B.F. Spencer Jr., Y. Suzuki (Eds.), US-Japan Workshop on Smart Structures for Improved Seismic Performance in Urban Regions, Seattle, WA, 2001, pp. 31-43.

[4] J.E. Doherty, Nondestructive evaluation, in: A.S. Kobayashi (Ed.), Handbook on Experimental Mechanics, Society for Experimental Mechanics, Englewood Cliffs, NJ, 1987 (Chapter 12).

[5] P.Z. Szewczyk, P. Hajela, Damage detection in structures based on features-sensitive neural networks, ASCE J. Comput. Civil Eng. 8 (2) (1994) 163-178.

[6] C. Zang, M. Imregun, Structural damage detection using artificial neural networks and measured FRF data reduced via principal component projection, J. Sound Vib. 242 (5) (2001) 813-827.

[7] S.S. Haykin, Neural Networks: A Comprehensive Foundation, Prentice-Hall, Upper Saddle River, NJ, 1999.

[8] S.F. Masri, T.K. Caughey, A nonparametric identification technique for nonlinear dynamic problems, J. Appl. Mech. 46 (1979) 433-445.
[9] K.S. Narendra, S. Mukhopadhyay, Neural networks for system identification, IFAC System Identification, Vol. 2, Kitakyushu, Fukuoka, Japan, 1997, pp. 735-744.

[10] S.F. Masi, A.G. Chassiakos, T.K. Caughey, Neural network approach to detection of changes in structural parameters, ASCE J. Eng. Mech. 122 (4) (1996) 350-360.

[11] S.-L. Hung, C.Y. Kao, Structural damage detection using the optimal weights of the approximating artificial neural networks, Earthquake Eng. Struct. Dyn. 31 (2002) 217-234.

[12] S. Saadat, G.D. Buckner, T. Furukawa, M.N. Noori, An Intelligent Parameter Varying (IPV) Approach for Non-linear System Identification of Base Excited Structures, Int. J. Nonlinear Mech. 39 (6) (2004) 993-1004.

[13] A. Masuda, A. Kakaoka, A. Sone, S. Yamamoto, Health monitoring system of structures based on orthonormal wavelet transform, Seismic Eng. 312 (1995) 161-167.

[14] Z. Hou, M. Noori, R. St. Amand, Wavelet-based approach for structural damage detection, ASCE J. Eng. Mech. 126 (7) (2000) 677-684.

[15] M. Mallat, A theory for multi-resolution signal decomposition: the wavelet representation, IEEE Pattern Anal. Mach. Intell. 11 (7) (1989) 659-674.

[16] J.W. Tedesco, W.G. McDougal, C.A. Ross, Structural Dynamics, Theory and Applications, Addison-Wesley Longman Inc., Menlo Park, CA, 1999.

[17] O. Nelles, Nonlinear System Identification: From Classical Approaches to Neural Networks and Fuzzy Models, Springer, New York, 2001. 